\title{
Investigation into Accurate Mass Capability of Matrix-Assisted Laser Desorption/Ionization Time-of-Flight Mass Spectrometry, with Respect to Radical Ion Species
}

\author{
Mark F. Wyatt, Bridget K. Stein, and A. Gareth Brenton \\ EPSRC National Mass Spectrometry Service Center, Department of Chemistry, University of Wales Swansea, \\ Swansea, United Kingdom
}

\begin{abstract}
Matrix-assisted laser desorption/ionization time-of-flight mass spectrometry (MALDITOFMS) has been shown to be an effective technique for the characterization of organometallic, coordination, and highly conjugated compounds. The preferred matrix is $2-[(2 E)-$ 3-(4-tert-butylphenyl)-2-methylprop-2-enylidene]malononitrile (DCTB), with radical ions observed. However, MALDI-TOFMS is generally not favored for accurate mass measurement. A specific method had to be developed for such compounds to assure the quality of our accurate mass results. Therefore, in this preliminary study, two methods of data acquisition, and both even-electron (EE + ) ion and odd-electron $(\mathrm{OE}+\cdot)$ radical ion mass calibration standards, have been investigated to establish the basic measurement technique. The benefit of this technique is demonstrated for a copper compound for which ions were observed by MALDI, but not by electrospray (ESI) or liquid secondary ion mass spectrometry (LSIMS); a mean mass accuracy error of -1.2 ppm was obtained. (J Am Soc Mass Spectrom 2006, 17, 672-675) ( 2006 American Society for Mass Spectrometry
\end{abstract}

$\mathrm{A}$ ccurate mass measurement is a very important application of mass spectrometry, allowing the elemental formulae of small molecules to be determined [1]. The number of possible formulae matching such a measurement decreases with greater precision, but increases with increasing mass and variety of elements, making identification more difficult [2, 3]. The background of accurate mass measurement is summarized elsewhere [4], and general operational guidelines are available [5]. Various mass spectrometers and ionization modes are used for accurate mass measurement, but matrix-assisted laser desorption/ionization time-of-flight mass spectrometry (MALDI-TOFMS) can have severe limitations, as accuracy can be compromised by poor peak shape. At Swansea, computerassisted peak matching on a magnetic sector instrument with an electrospray (ESI) source [6] is preferred for the wide range of samples received. Alternative sources are used where ESI is not appropriate, and while these systems generally give excellent results, some samples specifically require MALDI ionization.

The application of delayed extraction [7] to MALDI instrumentation, in conjunction with a reflectron ana-

Published online March 15, 2006

Address reprint requests to Dr. M. F. Wyatt, EPSRC National Mass Spectrometry Service Center (NMSSC), Department of Chemistry, University of Wales Swansea, Singleton Park, Swansea, West Glamorgan SA2 8PP, UK. E-mail: m.f.wyatt@swansea.ac.uk lyzer, afforded significant improvements in resolution and mass accuracy [8]. Subsequently, accurate mass measurements of peptides and proteins with internal standards have achieved errors of mass accuracy of $<5 \mathrm{ppm}[9,10]$. It may be a journal requirement for analyte identification that the accuracy of mass measurement be $<5$ ppm [11], and this general benchmark is widely used. Thus, it has already been demonstrated that MALDI-TOFMS is comparable with most techniques. However, it has been noted in a recent intercomparison study [4] that mass measurement errors can be $>10 \mathrm{ppm}$ where radical ion $(\mathrm{OE}+\cdot)$ species of an analyte were calibrated with sodiated poly(ethylene glycol) oligomers. Similar observations have been made within our laboratory, and are likely to be the result of the different ionization processes that produce each species.

Recently, we have shown that the aprotic matrix 2-[(2E)-3-(4-tert-butylphenyl)-2-methylprop-2-enylidene] malononitrile (DCTB) is a very effective matrix for several organometallic, coordination, and highly conjugated compounds, with $\mathrm{OE}+$. ions being observed [12]. Therefore, accurate mass measurement of these compounds via MALDI is a logical progression. Here, DCTB may have an advantage over traditional, acidic matrices, which often produce a mixture of $\mathrm{OE}+\cdot$ and protonated ion species with these compounds. These overlapping species are not resolved by a TOF analyzer, and, therefore, only the 
Table 1. Details of Compounds 1-6.

\begin{tabular}{|c|c|c|c|c|c|}
\hline Compound & Description & Measured isotopic species & Theoretical $\mathrm{m} / \mathrm{z}$ & Ref. & Ion parity \\
\hline 1 & $\begin{array}{l}\text { Gold-(polyethylene glycol (PEG) } \\
\text { carbene)-chloride }\end{array}$ & ${ }^{12} \mathrm{C}$ for each species & $\begin{array}{r}889.4020 \text { (9-mer) } \\
933.4283 \text { (10-mer) } \\
977.4545 \text { (11-mer) } \\
1021.4807 \text { (12-mer) }\end{array}$ & 13 & Even \\
\hline 2 & $\begin{array}{l}\text { Zinc-complexed ferrocene } \\
\text { porphyrin }\end{array}$ & ${ }^{12} \mathrm{C}_{58}{ }^{1} \mathrm{H}_{38}{ }^{14} \mathrm{~N}_{4}{ }^{56} \mathrm{Fe}^{64} \mathrm{Zn}$ & 910.1732 & 14 & Odd \\
\hline 3 & Ferrocene porphyrin & ${ }^{12} \mathrm{C}_{58}{ }^{1} \mathrm{H}_{40}{ }^{14} \mathrm{~N}_{4}{ }^{56} \mathrm{Fe}$ & 848.2598 & 14 & Odd \\
\hline 4 & $\begin{array}{l}\text { Neutral iridium coordination } \\
\text { compound }\end{array}$ & ${ }^{12} \mathrm{C}_{52}{ }^{1} \mathrm{H}_{47}{ }^{14} \mathrm{~N}_{2}{ }^{16} \mathrm{O}_{2}{ }^{32} \mathrm{~S}_{2}{ }^{193} \mathrm{Ir}$ & 988.2704 & 15 & Odd \\
\hline 5 & $\begin{array}{l}\text { Cationic copper coordination } \\
\text { compound (diperchlorate salt) }\end{array}$ & ${ }^{12} \mathrm{C}_{34}{ }^{1} \mathrm{H}_{24}{ }^{14} \mathrm{~N}_{6}{ }^{16} \mathrm{O}_{10}{ }^{63} \mathrm{Cu}_{2}{ }^{35} \mathrm{Cl}$ & 836.9829 & 16 & Even \\
\hline 6 & Triflated porphyrin & ${ }^{12} \mathrm{C}_{45}{ }^{1} \mathrm{H}_{29}{ }^{14} \mathrm{~N}_{4}{ }^{16} \mathrm{O}_{3}{ }^{19} \mathrm{~F}_{3}{ }^{32} \mathrm{~S}$ & 762.1907 & 14 & Odd \\
\hline
\end{tabular}

lowest mass of the isotopic distribution should be measured, the abundance of which may be insufficient. However, DCTB is not compatible with peptide standards normally used for calibration. Additionally, a deep-welled sample plate is advantageous due to the use of very volatile solvents, but this prevents a "close"-external calibration from being performed. Therefore, we are exploring the possibility of an accurate mass measurement protocol using similar compounds as internal standards, to achieve the best possible level of accuracy for mass measurement using MALDI-TOFMS, with $<5$ ppm error as our yardstick.

\section{Experimental}

Compounds 1-6 [13-16] (see Table 1) were submitted for analysis to the EPSRC National Mass Spectrometry Service Centre (NMSSC) [17], as part of the normal operation of the Service. DCTB matrix (highest purity available) was purchased from Fluka (Dorset, UK). MALDI-TOFMS spectra were acquired using an Applied Biosystems Voyager DE-STR spectrometer (Framingham, MA), which is equipped with a nitrogen laser. Data were baseline corrected, noise filtered, centroided at 50\%, and a two-point bracketing calibration performed, utilizing Data Explorer V4.0 software supplied by Applied Biosystems. Further experimental details are included in the Supplementary Material section, which can be found in the electronic version of this article.

The current study has three main parts:

1. Two methods of automated data acquisition were investigated; accumulation of 25 laser shots from two locations (Method A) and 50 shots from a single location (Method B), within a sample spot. This was accomplished with $\mathbf{1}$, which appears to undergo in-source dissociation upon irradiation, where

$$
2 \mathrm{ClAu}(\mathrm{PEG} \text {-carbene }) \rightarrow \mathrm{AuCl}_{2}^{-}+\mathrm{Au}(\mathrm{PEG} \text {-carbene })_{2}^{+}
$$

to give a distribution of even-electron $(\mathrm{EE}+)$ ion species covering the 700-1200 Da mass range. Results were generated for each method, using the 11-mer species as the analyte, bracketed by the 10and 12-mer species as calibration standards, (see Supplementary Material, Figure S1).

2. The investigation of whether analyte and standards need to have the same mechanism of ionization, e.g., whether $\mathrm{OE}+$. ion analytes should be calibrated with $\mathrm{OE}+\cdot$ ion standards. Compound 2 was chosen as the $\mathrm{OE}+$. ion analyte, with $\mathbf{3}$ and $\mathbf{4}$ chosen as the $\mathrm{OE}+\cdot$ ion calibration standards. The 9- and 10-mer species of $\mathbf{1}$ were chosen as $\mathrm{EE}+$ ion calibration standards (see Supplementary Material, Figure S2).

3. Case study of Compound 5, which had failed to ionize by any of our ionization methods other than MALDI. Calibration standards were 2 and $\mathbf{6}$.

\section{Results and Discussion}

\section{Method A Versus Method B}

To easily create a large sized dataset, data were acquired automatically, using the Sequence application on the Voyager. Parameters such as signal intensity were not fully optimized, hence there may be errors associated with automatic acquisition. Errors in mass accuracy for both of the acquisition methods are given in Figure 1. Descriptive statistics for both sets of results are presented in Table 2.

It is encouraging to note that the mean mass accuracy error for both methods was $<1 \mathrm{ppm}$, and that no result was $>10 \mathrm{ppm}$. The results were evaluated using statistical significance tests [18]. The paired $t$-test value is considerably less than the critical value $\left(t_{\mathrm{c}}=2.045\right.$, evaluated for $n-1$ (=29) degrees of freedom), indicating no significant difference between the mean error values. This indicates further that sample topography, including the relatively small separating distances between analyte and calibrant, might not be as important as thought previously. Similarly, the two-tailed F-test value, which compares the variances of datasets, is also considerably less than the critical value, indicating that neither method was more precise. A simple $t$-test can be used to evaluate systematic errors. The $t$-test value for Method A dataset, being noticeably less than the critical 


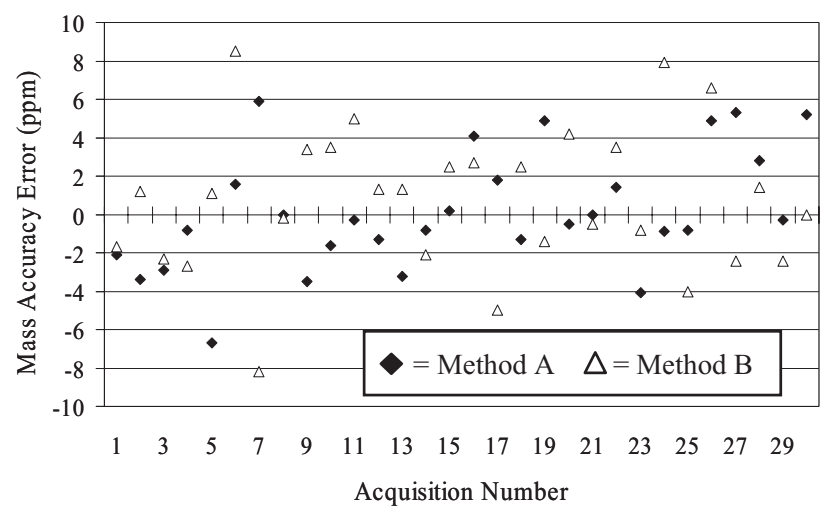

Figure 1. Plot of the errors in mass measurement accuracy (ppm) for EE+ ion, 1, and automated Methods A and B.

value, implies strongly that the method is free from systematic errors. However, for the Method B dataset, the calculated $t$-test and $t_{\mathrm{c}}$ values are very similar, so that any conclusions concerning evidence for systematic errors would have a high degree of uncertainty. This shift towards systematic error is likely to arise from a combination of minimum acceptable signal intensity and signal-to-noise ratio specified for automatic acquisition and poor peak shape. Analyte signals often decrease to zero within a few laser shots, with noise accumulated thereafter, which can result in poor peak shapes and thus affect mass accuracy. When trying to acquire data near the analyte signal threshold, it is often necessary to move around a sample spot to maintain observable signals. By raising the minimum acceptable signal intensity from 1000 to 5000, acceptable signal-tonoise ratios and peak shapes were observed for all three signals. Method A was used for the remainder of the study as there is a high degree of confidence that the method is free from systematic errors.

\section{EE+ Ion Versus OE+. Ion Standards}

Errors in mass accuracy for both types of calibration standard are given in the Supplementary Material, Figure S3. Descriptive statistics for both sets of results are presented in Table 3. Although an improvement in precision is observed, probably due to enhanced acquisition parameters, an increase in the mean error values

Table 2. Statistics for the errors in mass measurement accuracy (ppm) for EE+ ion, 1, and automated Methods A and B

\begin{tabular}{lccc}
\hline \multicolumn{1}{c}{ Statistic } & Method A & Method B \\
\hline \hline Mean error (ppm) & 0.1 & & 0.8 \\
Standard deviation (ppm) & 3.2 & 3.8 \\
Confidence level (ppm, 95\%) & 1.2 & & 1.4 \\
$t$-test & 0.370 & & 2.156 \\
$t_{\mathrm{c}(29)}(95 \%)$ & 2.045 & & 2.045 \\
Paired $t$-test & & 0.475 & \\
$t_{\mathrm{c}(29)}(95 \%)$ & 2.045 & \\
Two-tailed F-test & & 0.346 & \\
Two-tailed $\mathrm{F}_{\mathrm{c}(29,29)}(95 \%)$ & & 2.08 & \\
\hline
\end{tabular}

Table 3. Statistics for the errors in mass measurement accuracy (ppm) using EE+ ion, 1, and $\mathrm{OE}+\cdot$ ion standards, 3 and $\mathbf{4}$, and automated Method A

\begin{tabular}{lccc}
\hline \multicolumn{1}{c}{ Statistic } & EE + ions & OE $+\cdot$ ions \\
\hline \hline Mean error (ppm) & 4.5 & & 2.3 \\
Standard deviation (ppm) & 2.8 & & 2.9 \\
Confidence level (ppm, 95\%) & 1.1 & & 1.01 \\
$t$-test & 14.769 & & 7.294 \\
$t_{\mathrm{c}(29)}(95 \%)$ & 2.045 & & 2.045 \\
Paired $t$-test & & 0.004 & \\
$t_{\mathrm{c}(29)}$ (95\%) & & 2.045 & \\
Two-tailed F-test & & 0.852 & \\
Two-tailed $\mathrm{F}_{\mathrm{c}(29,29)}(95 \%)$ & & 2.08 & \\
\hline
\end{tabular}

for mass accuracy is also observed. While these mean values are still $<5 \mathrm{ppm}$, the $t$-test values are considerably greater than the critical value $\left(t_{\mathrm{c}}=2.045\right)$, indicating convincingly the presence of systematic errors for both datasets, which is apparent from the positive bias observed in each. However, the paired $t$-test value, being noticeably less than the critical value, suggests strongly that there is no significant difference between the mean values despite the mean error for the $\mathrm{EE}+$ ion standards being twice that for the $\mathrm{OE}+\cdot$ ion standards; neither species of calibrant is favored statistically. Similarly, the two-tailed F-test value, being noticeably less than the critical value, indicates strongly that neither method is more precise. The systematic errors arise from a number of factors, which include those particular sample preparations, the use of, and values given to, automatic acquisition parameters.

In practice, accurate mass measurements using MALDI are generally performed manually to ensure the best possible data quality. The samples were prepared again and measurements repeated manually (see Supplementary Material, Figure S4); statistics are given in Table 4 . The paired $t$-test and two-tailed F-test results were similar to before, so the conclusions drawn are unchanged. However, there was now no evidence for systematic errors in the mean errors of the measured masses, from the $t$-test for both datasets. Consequently, Method A, employed manually with either calibrant, appears to fulfill all necessary criteria to be viable for

Table 4. Statistics for the errors in mass measurement accuracy (ppm) using EE + ion, 1, and $\mathrm{OE}+\cdot$ ion standards, $\mathbf{3}$ and $\mathbf{4}$, and manual Method A

\begin{tabular}{lccc}
\hline \multicolumn{1}{c}{ Statistic } & EE + ions & OE + ions \\
\hline \hline Mean error (ppm) & 1.2 & & 0.5 \\
Standard deviation (ppm) & 1.7 & & 2.1 \\
Confidence level (ppm, 95\%) & 2.1 & & 2.6 \\
$t$-test & 0.208 & & 0.185 \\
$t_{\mathrm{c}(4)}(95 \%)$ & 2.776 & & 2.776 \\
Paired $t$-test & & 0.587 & \\
$t_{\mathrm{c}(4)}(95 \%)$ & & 2.776 & \\
Two-tailed F-test & 0.666 & \\
Two-tailed $\mathrm{F}_{\mathrm{c}(4,4)}(95 \%)$ & & 9.605 & \\
\hline
\end{tabular}


MALDI accurate mass measurement of organometallic, coordination, and highly conjugated compounds.

\section{Case Study}

Compound 5 was submitted to the NMSSC requesting both low-resolution and accurate mass analyses. The sample was a diperchlorate salt, but the expected $\left[\mathrm{M}-\mathrm{ClO}_{4}\right]^{+}(\mathrm{EE}+)$ ion was not observed in ESI or LSIMS. However, this ion was observed by MALDITOFMS, giving the opportunity to try the above method of accurate mass measurement on a real sample. The $\mathrm{Au}$ (PEG-carbene $)_{2}{ }^{+}$calibration standards were used initially, but the abundance of the lower mass standard peak was too low to give an acceptable signal, so $\mathrm{OE}+$. ion calibration standards $\mathbf{2}$ and $\mathbf{6}$ were employed. Errors in mass accuracy are given in the Supplementary Material, Figure S5. The mean mass accuracy error was $-1.2 \mathrm{ppm}$, the standard deviation was $2.8 \mathrm{ppm}$, and the $95 \%$ confidence level was $3.5 \mathrm{ppm}$. The method thus proved itself to be potentially useful for real samples.

\section{Conclusions}

This preliminary study has shown that, under appropriate conditions, accurate mass measurement with the benchmark accuracy of $<5 \mathrm{ppm}$ is achievable by MALDI-TOFMS. Acquisition of data using Method A appears to benefit mass accuracy. Manual data acquisition methodology seems to be good practice for avoiding certain systematic errors. Although this was not an aim of the study, for the compounds investigated, we observed that the presence or absence of metals appeared to have no effect on mass accuracy. Similarly, mass accuracy does not appear to be affected by whether or not the analyte and calibrant are the same type of ion species (EE+ or $\mathrm{OE}+\cdot$ ) for the compounds studied, which is in disagreement with results obtained using sodiated oligomers as calibrants for radical ions. This observation could be directly related to the dissociation process of $\mathbf{1}$. These species were a useful calibration standard over the mass range 890-1020 Da. Outside of this range, e.g., in the case of Compound 5, ion signals were not intense enough to be a good calibrant.

To continue this work, other calibration materials applicable to the majority of organometallic, coordination, and highly conjugated compounds, and preferably commercially available, need to be identified. Ideally, two oligomeric standards are desirable to cover the whole mass range over which MALDI accurate mass measurement is required for publication purposes, ca. 500-1500 Da. The standards should have different end-groups to avoid any mass overlap with the analyte.

\section{Acknowledgments}

The authors thank EPSRC (contract/grant number GR/R70088/ 01) for funding this work, the users of the NMSSC who kindly gave them permission to use their samples and mass spectrometry data for this publication, and their colleague Dr. A.P. Hunter, who acquired the initial MALDI-TOFMS data for 5.

\section{References}

1. Beynon, J. H. High Resolution Mass Spectrometry of Organic Materials. Adv. Mass Spectrom. 1959, 328-354.

2. Gross, M. L. Accurate Masses for Structure Confirmation. J. Am. Soc. Mass Spectrom. 1994, 5, 57-57.

3. Price, P. C.; Gale, P. J.; Loo, J. A.; Heller, D. N.; Richardson, S. D.; Duncan, M. W. Proceedings of the 50th ASMS Conference on Mass Spectrometry and Allied Topics; Orlando, FL, June, 2002.

4. Bristow, A. W. T.; Webb, K. S. Intercomparison Study on Accurate Mass Measurement of Small Molecules in Mass Spectrometry. J. Am. Soc. Mass Spectrom. 2003, 14, 1086-1098.

5. http://www.bmss.org.uk/Docs/VIMMS_guide.pdf.

6. D'Agostino, P. A.; Hancock, J. R.; Provost, L. R.; Semchuk, P. D.; Hodges, R. S. High-Resolution Electrospray Mass Spectrometry with a Magnetic-Sector Instrument Accurate Mass Measurement and Peptide Sequencing. Rapid Commun. Mass Spectrom. 1995, 9, 597-603.

7. Wiley, W. C.; Mclaren, I. H. Time-of-Flight Mass Spectrometer with Improved Resolution. Rev. Sci. Instrum. 1955, 26, 1150-1157.

8. Vestal, M. L.; Juhasz, P.; Martin, S. A. Delayed Extraction MatrixAssisted Laser-Desorption Time-of-Flight Mass-Spectrometry. Rapid Commun. Mass Spectrom. 1995, 9, 1044-1050.

9. Takach, E. J.; Hines, W. M.; Patterson, D. H.; Juhasz, P.; Falick, A. M.; Vestal, M. L.; Martin, S. A. Accurate Mass Measurements Using MALDI-TOF with Delayed Extraction. J. Protein Chem. 1997, 16, 363-369.

10. Edmondson, R. D.; Russell, D. H. Evaluation of Matrix-Assisted Laser Desorption Ionization Time-of-Flight Mass Measurement Accuracy by Using Delayed Extraction. J. Am. Soc. Mass Spectrom. 1996, 7, 995-1001.

11. Guidelines for Authors. J. Org. Chem. 2005, 70, 20A.

12. Wyatt, M. F.; Stein, B. K.; Brenton, A. G. Characterization of Various Analytes Using Matrix-Assisted Laser Desorption/Ionization Time-ofFlight Mass Spectrometry and 2-[(2E)-3-(4-tert-Butylphenyl)-2-Methylprop-2-Enylidene]Malononitrile Matrix. Anal. Chem. 2006, 78, 199-206.

13. Howell, J. A. S. Gold Layer Deposition. British Patent no. 0508242.5; 2005.

14. Cammidge, A. N.; Scaife, P. J.; Berber, G.; Hughes, D. L. Cofacial Porphyrin-Ferrocene Dyads and a New Class of Conjugated Porphyrin. Org. Lett. 2005, 7, 3413-3416.

15. Evans, N. R., Holmes, A. B. Sample submitted to the NMSSC; University of Cambridge, UK; unpublished.

16. Singh, N., Amoroso, A. J., Dervisi, A., Edwards, P. G., Ooi, L. Dalton Trans., unpublished.

17. http://www.swan.ac.uk/nmssc.

18. Miller, J. C.; Miller, J. N. In Statistics for Analytical Chemistry, 2nd ed.; Ellis Horward Ltd.: Chichester, England, 1988; pp 53-77. 